

\section{INDICE}

\section{ÁMBITOS PERSONALES PERSONAL ÁMBITOS}

Las revistas satíricas sevillanas en el primer tercio del siglo XX y sus dibujantes

Sevillian satirical magazines in the first third of the 20th century and their cartoonists

J. Carlos Méndez Paguillo

\section{ARTÍCULOS ARTICLES}

El humor en la publicidad radiofónica. La marca de la Cadena SER a través de las promos de Ortega Humour in radio advertising. The brand of the Cadena SER through the Ortega's advertisements

Javier de Sola Pueyo, Ana Segura Anaya, Antonia Isabel Nogales-Bocio

La narrativa transmedia en la publicidad: el caso de "LEGO"

Transmedia narrative in advertising: the case of "LEGO"

Jaime Humberto Caldera Chacón, Gloria Olivia Rodríguez Garay

Nuevos relatos híbridos en el cine de ficción español. El caso de Entre dos aguas de Isaki Lacuesta New hybrid stories in Spanish fiction cinema. The case of Entre dos aguas by Isaki Lacuesta

Manuel Blanco Pérez

$60-73$

La serie de TV 'Medici': Entre historia, memoria y producto comercial

The TV series 'Medici': between history, memory and comercial product

Pamela Giorgi, Irene Zoppi

Realidad y ficción en el discurso informativo. Crímenes como inspiración para proyectos audiovisuales en España Reality and fiction in informative discourse. Crimes as inspiration for audiovisual projects in Spain 
Relatório Macbride: Releitura à luz de ameaças ao direito à comunicação nas plataformas digitais MacBride report: re-reading in light of threats to the right to communication on digital platforms Lilian Bartira Santos Silva, Carla Azevedo de Aragão, Nelson De Luca Pretto

La violencia de género en Twitter según Vox en las elecciones autonómicas de Andalucía Gender violence on Twitter according to Vox in the regional elections of Andalusia Sergio Luque Ortiz, Mónica Cano Alarcón 116-133 Estructura mediática china: una aproximación al caso de China Central Television (CCTV) Chinese media structure: an approximation to the case of China's Central Television (CCTV) 


\title{
Estructura mediática china: una aproximación al caso de China Central Television (CCTV)
}

\author{
Chinese media structure: an approximation to the case of China's Central \\ Television (CCTV)
}

\author{
Lucía Varela Monterroso \\ Universidad Carlos III | Calle Madrid, 126 (edificio Decanato), 28903 Getafe (Madrid) | España | \\ https://orcid.org/0000-0003-3150-8651 | luciavarelamonterroso@gmail.com
}

Fechas | Recepción: 22/10/2020 | Aceptación: 19/11/2020 | Publicación final: 15/01/2021

\begin{abstract}
Resumen
La superpotencia emergente China se ha convertido en los últimos años en una pieza fundamental en el tablero informativo mundial. Este estudio pretende ahondar en la estructura mediática china, centrando especial atención en el grupo televisivo público China Central Television. En 2018 el gobierno chino lleva a cabo un "Plan de Reforma Institucional estatal y del Partido de Profundización", cuyo objetivo principal se basa en la mejora de la opinión pública sobre China a escala mundial. Para alcanzar este objetivo, se creó el conglomerado mediático transnacional China Media Group. Este se ha segmentado y, actualmente, controla tres gigantes mediáticos chinos: a nivel televisivo con China Central television (CCTV) y a nivel radiofónico con China National Radio (CNR) y China Radio International (CRI). Mediante una metodología historiográfica y la puesta en práctica de un enfoque metodológico descriptivo se profundizará en los siguientes objetivos. En primer lugar, se busca vislumbrar la forma de organización de los medios de comunicación chinos. Seguidamente se pretende conocer la forma de control llevada
\end{abstract}

\begin{abstract}
China's Emerging superpower has become a key piece on the global information board in recent years. This study aims to delve into the Chinese media structure, paying special attention on the public television group China Central Television. From a diacritical perspective, one seeks to understand the crossroads underlying it; a descriptive methodological approach focused on content analysis will take an in-depth look at how the media and the administration that control them will be controlled. In 2018, the Chinese government carried out a "State institutional reform plan and the Deepening Party" whose main objective is based on improving public opinion about China on a global scale.

Therefore, the transnational media conglomerate China Media Group was created. It has segmented and currently controls three Chinese media giants: on a television level, with China Central Television (CCTV) and radio with China National Radio and China Radio International. Using a historiographic methodology and the implementation of a descriptive methodological
\end{abstract}

Forma de citar:

Varela Monterroso , L. (2021). Estructura mediática china: una aproximación al caso de China Central Television (CCTV). Ámbitos. Revista Internacional de Comunicación, 51, pp.134-149. doi: 10.12795/Ambitos.2020.i51.09 
a cabo por el gobierno sobre la información y los medios de comunicación y, finalmente, se abordará el contenido temático de la televisión pública china (CCTV). Cobra especial relevancia las ansias de expansión del gobierno chino a todo el mundo, el cual aspira a exportar las singularidades chinas $y$, por consiguiente, a convertirse en un contrapunto al control único ejercido actualmente por Estados Unidos a nivel mundial. La comunicación es para China un punto clave de esta expansión.

Palabras clave: China media group; China Central Television; control; televisión approach we will deepen into the following objectives. First, we seek to glimpse the way Chinese media organization is. It is then when we intended to know the way of control carried out by the government related to information and media and will eventually address the thematic content of Chinese public television (CCTV). The Chinese government's desire for expansion around the world is particularly important, which aims to export Chinese singularities and thus to become a counterpoint to the single control currently exercise by the United States globally. Communication is a key point for China in this expansion.

Keywords: China media group; China Central Television; regulation; television

\section{INTRODUCCIÓN}

Tras la proclamación por parte de Mao Zedong en 1949 de la República Popular de China, la comunicación ha pasado a ser controlada férreamente por el Estado. Desde entonces, hasta la actualidad, el Partido Comunista Chino (PCCh) ha controlado todos los niveles del Estado, entre ellos la comunicación.

En 1978, tras la muerte de Mao Zedong, llega al poder Deng Xiaoping heredando un Estado en su mayoría pobre. Para paliar esta situación fomenta las conocidas como "cuatro modernizaciones", cuyo pilar fundamental se sustenta en la búsqueda de protagonismo en el mercado a nivel mundial. Sumado a estas cuatro modernizaciones impulsadas por Deng, el ámbito de la comunicación cobró un limitado aperturismo donde los mass media, caracterizados por una falta de transparencia y un férreo control estatal, pasaron a depender no tanto de la financiación pública, sino también de los contenidos publicitarios.

Este "menor control" experimentado tras el cambio de gobierno no alteró de forma sustancial la estructuración interna de la comunicación; actualmente sigue funcionando la existencia de un complicado entramado institucional encargado de controlar la información. (Ríos, 2016).

Los medios de comunicación al estar bajo el mandato del propio Estado se han convertido en una forma de extensión de propaganda institucional y de entretenimiento a la población donde las críticas al PCCh, y por lo tanto al gobierno del país, son cada vez más limitadas. 
Las ansias de expansión no frenaron a este gigante, para conseguir mayor influencia a nivel mundial se lleva a cabo la traducción de los contenidos tanto informativos como de entretenimiento, alcanzando así un mayor número de audiencia a nivel global.

En esta investigación se plantean los siguientes objetivos:

10 Objetivo: Vislumbrar la forma organizativa de los medios de comunicación en China y la relación subyacente de los mismos con el poder.

2ㅇ Objetivo: Conocer la forma de control llevada a cabo por el gobierno y las administraciones encargadas de la regulación y vigilancia de los medios de comunicación.

3o Objetivo: Analizar el conglomerado mediático China Media Group centrándose en el análisis de la televisión pública china, CCTV.

4o Objetivo: Analizar la forma de expansión comunicativa de China a nivel mundial.

Partiendo de los objetivos anteriores se establecen las siguientes hipótesis:

10 Hipótesis: El gobierno chino realiza un control férreo sobre la comunicación tanto a nivel estatal como a la hora de filtrar, en ambas direcciones, los contenidos informativos del exterior.

20 Hipótesis: Este país asiático busca convertirse en un actor principal en el mundo actual, no solo a nivel económico sino también a nivel comunicativo.

3ํ Hipótesis: Los contenidos informativos chinos se desarrollan y traducen acorde a la zona donde vayan a ser emitidos siendo una clara señal de la intención de China de llegar a todas las partes del mundo.

Para entender en profundidad las encrucijadas que supone el estudio de la estructura mediática china es necesario elaborar un análisis partiendo de la diacronía de la misma. Es necesario poseer una aproximación de China, no solo a nivel comunicativo sino también a nivel global, donde cobra importancia el contexto político-institucional, social, económico y tecnológico que componen lo que es el país y todas sus relaciones e influencia alrededor del mundo. Una visión estática limitaría el acceso a la estructura sustancial de la misma ya que depende de múltiples factores externos. La metodología de investigación que se llevará a cabo parte del análisis del contenido desde un punto de vista descriptivo y un enfoque conceptual, de esta forma permitirá un estudio, lo más pormenorizado posible, de la estructura mediática del país.

Para ello se procederá a la lectura de informes y proyectos especializados tanto en la estructura mediática china como en la forma de organización del país y, por ende, del conjunto social. De esta manera se conseguirá una perspectiva global sobre el tema a tratar. La lectura y el análisis de las normativas que regulan los medios de comunicación chinos y la ley de derechos de propiedad permitirán conocer la influencia de potencias extranjeras en el mercado chino. De esta manera se buscará dar a conocer el estado actual de la estructura mediática china, teniendo en cuenta su influencia a lo largo del tiempo. Además, 
se analizará y se concretará los principales productos ofrecidos por CCTV en el que se examinará cuál es la temática predominante.

Es importante destacar la ausencia general de trabajos que analicen en profundidad y no un análisis somero de la estructura mediática; además la falta de información verídica y de atribución directa a la fuente complica la investigación en este proyecto. El problema reside en que el gobierno chino no pone a libre disposición las normas y regulaciones que metodizan la actividad informativa; estas normativas no están disponibles de forma directa, sino que es necesario acudir a terceros para conocer parte de estas, nunca su totalidad.

\section{ESTRUCTURA MEDIÁTICA CHINA}

\subsection{Contexto mediático}

La Constitución china recoge una serie de libertades y derechos en referencia a los medios de comunicación tal y como es la libertad de prensa o la libertad de expresión. El desarrollo de estos derechos implica una discordancia práctica debido a que las ideas que se muestren excesivamente críticas contra el Estado, y en consecuencia contra el PCCh, que puedan afectar a la práctica común de la sociedad son censuradas (Beina y Eleanor, 2017, p. 11).

Para analizar el proceso de transformación llevado a cabo por el sistema de comunicación chino es necesario conocer las variaciones que se produjeron en el mismo desde la instauración de la República Popular de China en 1949. Estos cambios afectaron de forma directa al conjunto de la sociedad y por consiguiente a la naturaleza de los medios de comunicación. La expansión de China, tanto a nivel internacional como nacional, ponen en evidencia la consolidación del liderazgo del PCCh.

Es relevante tener en cuenta la estrecha relación que tiene la comunicación a la hora de influir en determinados aspectos de la sociedad (Mancinas-Chávez, 2016, p.49-50).

La información afecta directamente en la forma de pensar y de actuar por parte de la sociedad, por tanto, el control de los medios de comunicación por parte del poder supone un control equidistante de la sociedad. Comunicación y poder tienen una relación directa (Salazar Navarro, 2012, p. 3).

El Estado chino, y por ende el PCCh, se ha adaptado de forma continua a los retos sociales y mundiales que se les han presentado a lo largo de los años llevando a cabo un control en la comunicación y en la hegemonía del poder mediante la aplicación de una política comunicativa asertiva.

Desde la fundación de la República Popular de China la comunicación se basa en un modelo vertical. En este momento de la historia, los medios de comunicación chinos orientaron sus esfuerzos a garantizar la propagación de una comunicación no agresiva a favor de los intereses del propio Estado. El papel protagonista del Estado en el control de los medios de comunicación supuso, de forma directa y a corto plazo, tensiones de los diversos medios con el propio gobierno. Esto fue debido a la instauración de una serie de medidas estatales para controlar qué se comunicaba. Esta situación trajo consigo un duro golpe para la prensa donde el periodismo independiente prácticamente desapareció, un ejemplo es la limitación de las temáticas que se podían incluir en la agenda setting (Salazar Navarro, 2012). 
Tras la muerte de Mao y el ascenso al poder de Deng, los medios de comunicación tienen que hacer frente a nuevas condiciones que modifican la actuación práctica de los mismos tras la aplicación de las "cuatro modernizaciones". El "socialismo chino" permite una nueva política donde se otorga, en cierta medida, voz al pueblo y se ofrece un mayor espacio a la crítica siempre y cuando esta no ponga en duda las bases del gobierno chino y del Estado (Salazar Navarro, 2012, p.14).

La fluctuación del sistema de mercado de la época supuso la entrada paulatina de pluralidad informativa, el acceso a la publicidad marcó un punto de inflexión en la práctica de los medios de comunicación donde aflora la posibilidad de buscar financiamiento mediante la comercialización del producto periodístico. Esta situación supuso que los mass media no estén bajo el amparo directo de las ayudas gubernamentales chinas, pero esto no implica que no se tengan que someter a la censura.

En las tres últimas décadas se ha llevado a cabo una serie de cambios relevantes en el sistema comunicativo. La apertura iniciada por Deng supuso el punto de partida a la expansión de un sistema comunicativo, este adquirió una mayor relevancia con la entrada de China en la Organización Mundial del Comercio en 2001 donde se adoptó una economía más liberalizada en relación con los mercados exteriores. Esta apertura del mercado supuso por ende una expansión de los sistemas de comunicación y telecomunicaciones.

El PCCh se caracteriza por una gran adaptación a los cambios en su entorno, la apertura de China a nivel mundial supuso una puerta de entrada para diferentes valores e informaciones que dificultan en gran medida el control ejercido por el Estado a lo largo de los años. Pero el posible espacio para la crítica política ha supuesto un punto relevante para el PCCh, cuyo objetivo se centra en conseguir el menor impacto posible de este tipo de informaciones negativas. Como señala Aira (como se cita en Cabacés, 2009), la figura del spin doctor es un individuo, perteneciente al gabinete de comunicación del partido, que intenta influir en la opinión pública colocando un sesgo favorable a la información que se le presenta al público. De esta forma se reorienta el mensaje amortiguando el impacto político negativo en la sociedad mediante una crítica interna. Esta técnica permite incapacitar los mensajes de terceros que buscan desestabilizar al poder con este tipo de informaciones peyorativas.

Hasta el año 2004 las actividades de producción y gestión de radio y televisión de compañías de capital extranjero estaban restringidas. A partir de 2007 la Asamblea Nacional Popular de China emite la Primera Ley sobre el Derecho de la Propiedad la cual prohíbe la participación externa en este tipo de actividades sin que un socio chino tenga un porcentaje accionarial mayoritario. Begoña de Suso (2007), abogada de la oficina de Shanghai, determina que esta regulación constituye un marco jurídico más seguro para la iniciativa privada, influyendo directamente en las actividades llevadas a cabo por los medios de comunicación cuya parte de su propiedad está en manos de empresas extranjeras. Este acontecimiento sumado a la entrada de China en la OMC provocó un mayor aperturismo del mercado comunicativo.

Mediante la fundación de administraciones, supeditadas a la vigilancia del Comité Central, el Estado controla de forma directa la información ejerciendo un control y tutela de los medios de comunicación. 


\subsection{Forma de control de la comunicación por parte del Estado chino}

El gobierno chino no ha fomentado una ley que regule la actividad periodística en el país a pesar de que en 1980 el Comité Central aprobó la puesta en marcha la redacción de una normativa de prensa, esta elaboración se paralizó debido a las manifestaciones derivadas en 1989 en la plaza de Tiananmen.

Desde entonces diversas voces de la sociedad han mostrado la necesidad de la elaboración de una ley para la práctica de la actividad periodística; a pesar de la inexistencia de este tipo de leyes se han elaborado diferentes Reglamentos de Administración que regulan diferentes campos como el de los productos audiovisuales.

La estructura de poder en China ha llevado a cabo mecanismos para el control del flujo de la información y la forma de transmitirla a la sociedad. Este sistema comunicativo tiene muy presente la importancia de su propia cultura, de la cual subyacen determinados valores afectando a la estructura tanto social, política o económica; estos valores influyen en gran medida a la hora de elaborar informaciones por parte de un medio de comunicación.

Para controlar la emisión de las noticias el Estado chino, y por consiguiente el PCCh, ha creado una telaraña de intereses, término acuñado por Ramón Reig (2010), en la que se conecta de forma directa la administración con los medios de comunicación. El Estado y el PCCh crearon administraciones propias dedicadas por completo al control de la información y el flujo de la misma; el objetivo principal fue el mantenimiento del statu quo en el país asiático.

Tal y como se ha mencionado anteriormente, la comunicación y el control de esta está ligado al propio PCCh. En el seno de la cúpula del poder chino subyace la figura del Politburó encargado de establecer el liderazgo de la información, llevar a cabo métodos de regulación de la comunicación y la creación de administraciones específicas encargadas de los órganos de propaganda (Madrid Morales et al ,2017).

Siguiendo las ideas iniciales de este proyecto, los medios de comunicación dependen de la intervención estatal, a su vez estos pueden poseer una dependencia financiera con el Estado de forma directa, indirecta o nula, siendo estos los medios de comunicación más independientes, pero a la vez son minoritarios y casi inexistentes.

\subsubsection{Normativas y organismos administrativos de control informativo)}

El control que realiza China en los mass media parte de los niveles más altos. En este punto se establece una relación directa del poder con los profesionales donde el propio gobierno chino asegurando así el alineamiento generalizado de los mass media. Los directores de los medios de comunicación son elegidos por el Comité y además se lleva a cabo un examen para poder ejercer la actividad periodística en el país (Madrid Morales et al, 2017).

Un organismo que es necesario mencionar en este punto es el Departamento de Propaganda del PCCh, el cual regula la información que se publica en los medios de comunicación. Se funda también el Grupo Central de Liderazgo para el Trabajo Ideológico y de Propaganda y el Comité Central para la Construcción de la Civilización Espiritual. Estos departamentos tienen como objetivo una efectiva supervisión del sistema de propaganda dirigido a todos los ámbitos de la sociedad china. Bajo la 
supervisión de estos tres órganos reguladores pertenecientes a la estructura del propio Estado se funda la Oficina de Información del Consejo de Estado, encargada de llevar un control sobre el estamento periodístico mediante un control a priori.

Los pilares en los que se sustenta la actividad de control sobre los medios de comunicación son los siguientes.

Por un lado, la Administración General de Prensa y Publicaciones (GAPP) está encargada del control y la monitorización de los medios de comunicación escritos, abarcando tanto impresos como electrónicos. Esta agencia es la que desempeña el control de la información a priori; entre sus funciones destaca en primer lugar la autorización de emitir las licencias de publicación. Una característica indispensable para conseguir esta licencia es que el medio de comunicación que la solicite debe estar respaldado por una institución oficial china; responsabilizándose así de la línea editorial del medio. Además, la GAPP administra los derechos de propiedad intelectual y emite los carnés de periodistas, obligatorios para ejercer la actividad periodística en el país.

Por otro lado, la Administración Estatal de Radio, Cine y Televisión (SARFT) es la apoderada en la vigilancia de los medios audiovisuales y ejerce un control directo sobre los mismos. Principalmente controla los servicios públicos oficiales de radio exterior (CRI-CNR), así como de la emisión de licencias orientadas tanto a la radio y a la televisión (CCTV) como a la industria del cine.

En 2013, tras la Asamblea Popular Nacional, el gobierno chino decide la unificación de estas dos piezas claves en el control de la comunicación. De esta forma se busca reducir y centralizar tanto las autorizaciones de los medios de comunicación como la supervisión de los mismos debido al gran auge de los medios tras la globalización y el acceso a internet. Con la fusión de la GAPP y la SARFT da lugar a la Administración Estatal de Prensa, Publicaciones, Radio, Cine y Televisión (SAPPRFT) en la que se busca reforzar el control y el liderazgo político del PCCH. Pero todo cambia en el XII Congreso Nacional del Pueblo de China, celebrado en marzo de 2018, donde se da luz verde a una reestructuración de la comunicación que provoca el cierre de la SAPPRFT y establece un nuevo mecanismo de regulación. Esta reforma institucional tiene como resultado directo la creación de tres administraciones estatales cuyas actividades estarán diferenciadas.

En primer lugar, la Administración Estatal de Cine es la encargada de supervisar las actividades de producción, distribución y proyección del producto cinematográfico y se asegura a su vez de que estas sigan los intereses estatales.

Seguidamente la Administración de Publicaciones, la cual funciona como la Administración Nacional de Derechos de Autor, es la encargada de la regulación, control y distribución de las noticias tanto escritas como virtuales.

Estas dos administraciones están bajo la vigilancia del Departamento de Publicidad del Comité Central del PCCh, encabezado por Huang Kunming que es miembro del Buró Político del Comité Central del PCCh y jefe del Departamento de Publicidad mencionado anteriormente. 
Por último, la Administración Estatal de Radio y Televisión estará controlada por el Consejo de Estado. Esta Administración se encarga de la elaboración de las políticas pertinentes para la gestión de los productos televisivos y radiofónicos, así como su implementación y desarrollo.

Esta reestructuración de la SAPPRFT, tras tan solo cinco años, se debe a la falta de eficacia en la funcionalidad de la organización. Es importante remarcar que la eliminación de la SAPPRFT no implica la eliminación de la censura en China, sino que establece los diferentes canales por los que se llevará a cabo este control gubernamental.

\subsubsection{Transmisión global de valores chinos}

La globalización ha conllevado la instauración por parte del Estado de una cimentación de los valores chinos y la transmisión de su identidad cultural tanto a nivel nacional como internacional. Esta distribución de creencias y valores se materializan por diferentes canales como por ejemplo la emisión de los contenidos mediáticos, tanto de entretenimiento como de información, conformando así la ideología del propio sistema. Claro está que los sistemas mediáticos poseen una gran influencia en la sociedad referente tanto a su forma de comportamiento como en su concepción social; debido a este motivo el poder está ligado con los medios de comunicación ya que este busca controlar el mensaje y por consiguiente tener un gran peso en la forma de pensar de la población.

Es importante tener presente la idea de que no todos los medios de comunicación chinos dependen de forma directa de la figura estatal, sino que el control se ha adaptado con el paso de los años manteniendo una vigilancia indirecta. La mayor parte de la información que se transmite en el país pasa por un proceso de autocensura por parte de los propios medios de comunicación, debido a la figura autoritaria del PCCh.

\section{TRANSFORMACIÓN MEDIÁTICA DE CHINA RADIO AND TELEVISION TRANSMISSION NETWORK CORPORATION A CHINA MEDIA GROUP}

En el año 2000 el gobierno chino inicia una estrategia comunicativa conocida como Going-Out ${ }^{1}$ que consiste en dotar a las empresas chinas de medios y recursos necesarios para conseguir su desarrollo en mercados extranjeros. En lo referente a la comunicación, esta estrategia se adaptó en busca de la creación de grupos mediáticos eficientes a nivel nacional y que estos a su vez que tengan un cuerpo y una capacidad de competir internacionalmente con otros conglomerados mediáticos. Se centró especial atención en Europa y América del Norte.

De esta forma el gobierno busca mitigar la imagen amenazante asociada internacionalmente a China y a su vez es un intento de reforzar la cohesión e identidad de la población del propio país basándose en el ideal confuciano de armonía.

La expansión internacional de la información china se llevó a cabo mediante un estudio previo de mercado; para ello, se estudió la historia, la política, la economía, la propia cultura del país y la audiencia para así ofrecer un mensaje lo más en consonancia posible con los de los países donde se

1 Cunhai, G. (2017). La imagen de China en América Latina en el siglo XXI. Orientando Temas de Asia Oriental, Sociedad, Cultura y Economía,14, de https://bit.ly/2HeAdUF . 
emitirá la programación china. De esta manera China busca cimentar las bases de una colaboración a corto, medio y largo plazo con países extranjeros (Cunhai, 2017).

Generalmente la influencia de los conglomerados mediáticos chinos no tiene un gran peso a nivel internacional, esta situación refleja un problema estructural caracterizado por la descentralización y la regionalización de los medios de comunicación del país. En este sentido, cobra relevancia la figura antagónica del conglomerado mediático China Media Group, fundado en 2018, tras el XII Congreso Nacional del Pueblo de China. China Media Group tiene presencia a nivel mundial gracias a una reestructuración interna con la integración de Central Television (CCTV), China National Radio (CNR) y China Radio International (CRI), que anteriormente formaban parte del conglomerado CRFTG.

Es relevante el nombre con el que se conoce a este conglomerado mediático, llamado popularmente Voice of China. Muestra un cierto contrapunto a Estados Unidos debido a que adquiere el mismo nombre de la emisora financiada por el gobierno llamada Voice of America. Se puede establecer esta coincidencia como una especie de guiño a la futura imagen que quiere conseguir China a nivel mundial mediante la emisión de contenidos chinos, siendo esta su voz, y proyectando a su vez una imagen positiva de este país asiático en el extranjero. De esta manera, este país asiático abre una propia ventana para garantizar que su propia voz se escuche a lo largo y ancho del territorio mundial; esta situación sumada a la traducción de los contenidos radiofónicos y televisivos muestran las ansias de expansión de China y además la capacidad de adaptación del gobierno a las circunstancias cambiantes. ${ }^{2}$

\section{CHINA CENTRAL TELEVISION}

A continuación, se analiza en concreto la televisión pública China Central Television (CCTV) como un acercamiento al conocimiento y análisis de la estructura mediática de este país asiático y su relación con el poder, siendo este un claro ejemplo de estrecha colaboración entre ambos organismos.

China Central Television (CCTV) es la mayor empresa audiovisual del país, un elemento mediático perteneciente al grupo estatal de comunicación China Media Group, siendo este un elemento empresarial. A su vez, su actividad depende del PCCh, el cual es un elemento político, que determina la línea editorial del propio medio y repercute en el mensaje ofrecido. Este mensaje se transmite a la sociedad haciendo referencia a la superestructura con la creación de valores y la identidad cultural de la población china.

La propia ideología del sistema, la cual conforma la superestructura, no solo se ofrece por la difusión de los medios de comunicación a través de mensajes persuasivos presentes tanto en el entretenimiento como en la espectacularización, sino que además esta ideología está intrínseca en la propia educación o viene dada en los mensajes publicitarios (Reig, 2017).

La difusión de informaciones por parte de CCTV a lo largo de los años se orientó en todo momento a una línea ideológica oficialista de los intereses del PCCh combinando estas emisiones con otras de carácter publicitario y de entretenimiento. Las opciones de entretenimiento y de difusión de poder

2 Guía de la Radio. (2018, 22 marzo). La República Popular China fusiona su radio y TV públicas en «Voice of China». Recuperado de https://bit.ly/2liGyio 
blando implican que la televisión, a partir de los años 90, se convirtiese en el "emblema del socialismo en el mercado chino" (Madrid Morales, et al 2017).

A partir de 1992 comienza la "era de oro de la CCTV" caracterizada por cambios tanto en la estructura interna del propio medio como de forma externa en la sociedad china debido a la creación de nuevas formas de ocio gracias a la televisión (Nelson, 2013).

Poco a poco el PCCH se fue adaptando a los cambios producidos en la sociedad emitiendo programas televisivos caracterizados por una visión más crítica a nivel social y gubernamental con la elaboración de reportajes de investigación o documentales orientados al ámbito social. Es importante tener en cuenta que la emisión de este tipo de programas más críticos con el gobierno parte de la decisión del poder central dotando a CCTV de un mayor margen de maniobra en su programación. Por lo tanto, el PCCh con la puesta en marcha de este tipo de contenidos transmite a la sociedad una imagen más pluralista de los medios de comunicación, donde se ofrece un espacio para el debate político y la crítica moderada a la gestión de gobierno. Es posible determinar que esto forma parte de una estrategia discursiva en la que se busca mostrar pluralidad e independencia por parte de la televisión estatal a pesar de que esta esté controlada de forma directa por el propio Partido.

Tabla 1 Canales CCTV

Los canales televisivos chinos existentes hasta la actualidad y su correspondiente temática

\begin{tabular}{|c|c|}
\hline Nombre del canal & Temática de la emisión \\
\hline CCTV-1 & Información generalista \\
\hline CCTV-2 & Canal económico y de finanzas \\
\hline CCTV-3 & Canal de arte y música \\
\hline CCTV-4 & \begin{tabular}{|l} 
Internacional. \\
$-\quad$ Posee canal de video en streaming en internet \\
$-\quad$ Retransmisión en chino mandarín \\
\end{tabular} \\
\hline CCTV- 4K & \begin{tabular}{|l}
$\mid$ Internacional \\
$-\quad$ Transmisión en alta definición
\end{tabular} \\
\hline CCTV-5 & Canal de deportes \\
\hline CCTV-5+ & \begin{tabular}{|l} 
Eventos deportivos \\
$-\quad$ Transmisión en alta definición
\end{tabular} \\
\hline CCTV-6 & \begin{tabular}{|l} 
Películas \\
- \\
- Centra su atención especialmente en cine nacional y sur- \\
- \\
coreano \\
Emite películas internacionales
\end{tabular} \\
\hline CCTV-7 & Defensa Nacional y Militar \\
\hline CCTV-8 & Series de Televisión y espectáculo \\
\hline CCTV-9 & \begin{tabular}{|l} 
Documental \\
$-\quad$ Transmisión en inglés
\end{tabular} \\
\hline CCTV-10 & Ciencia y educación \\
\hline CCTV-11 & Ópera y teatro chino \\
\hline
\end{tabular}




\begin{tabular}{|c|c|}
\hline CCTV-12 & Sociedad y derecho \\
\hline CCTV-13 & $\begin{array}{l}\text { News } 24 \text { horas } \\
-\quad \text { Transmisión en chino mandarín }\end{array}$ \\
\hline CCTV-14 & Programación orientada a los niños \\
\hline CCTV-15 & Música \\
\hline CCTV-16 & Juegos Olímpicos \\
\hline CCTV-17 & Agrícola y rural \\
\hline CGTN (News) & $\begin{array}{l}\text { Canal internacional de noticias } 24 \text { horas } \\
\text { - Transmisión en inglés }\end{array}$ \\
\hline CGTN-Español & $\begin{array}{l}\text { Noticias internacionales y entretenimiento } \\
-\quad \text { Canal en español. }\end{array}$ \\
\hline CGTN-Français & $\begin{array}{l}\text { Noticias internacionales y entretenimiento } \\
\text { - } \quad \text { Canal en francés }\end{array}$ \\
\hline 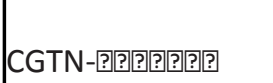 & $\begin{array}{l}\text { Noticias internacionales y entretenimiento } \\
-\quad \text { Canal en árabe }\end{array}$ \\
\hline CGTN-Русский & $\begin{array}{l}\text { Noticias internacionales y entretenimiento } \\
-\quad \text { Canal en ruso }\end{array}$ \\
\hline Documental CGTN & $\begin{array}{l}\text { Documental } \\
-\quad \text { Transmisión en inglés }\end{array}$ \\
\hline CGTN Asia & $\begin{array}{l}\text { Internacional. Dirigido a Asia y Australia } \\
\text { - Transmisión en inglés }\end{array}$ \\
\hline CGTN África & Internacional. Retransmisión en inglés \\
\hline CGTN América & $\begin{array}{l}\text { Internacional. Dirigido a América del Norte y América Latina } \\
\text { - Transmisión en inglés }\end{array}$ \\
\hline CGTN Europa & $\begin{array}{c}\text { Internacional. Dirigido a Europa } \\
\text { - Transmisión en inglés }\end{array}$ \\
\hline
\end{tabular}

Fuente: elaboración propia con datos extraídos de https://www.cctv.com

Tal y como se muestra en la tabla anterior, el Estado chino ha centrado especial atención y recursos en referencia a la expansión de la difusión internacional de su contenido de información y entretenimiento por parte de su grupo de comunicación estatal. Para ello ha elaborado cadenas dedicadas exclusivamente a la información en estos países donde los contenidos se adaptan según la audiencia. La adaptación estratégica de CCTV se caracteriza por la creación de una red de transmisión orientada a varios idiomas, debido a la limitación del conocimiento del chino mandarín a nivel mundial. El PCCh decidió comenzar a emitir contenidos en inglés para alcanzar así un mayor número de consumidores a nivel global, centrado en un primer lugar en América del Norte y Europa Occidental.

Actualmente esta corporación televisiva cuenta con 42 canales de televisión, de los cuales 29 son canales en abierto y los 13 restantes forman parte de canales digitales de pago.

En el año 2016, las operaciones internacionales de CCTV se renombraron como China Global Television Network y las relaciones de CCTV con otros países se comenzaron a realizar mediante la filial China International Television Corporation (CITVC). La función de este organismo es establecer acuerdos internacionales con otros grupos mediáticos de comunicación, logrando así un intercambio de 
contenidos de ambos países. De esta forma no solo el país asiático tiene presencia fuera de sus fronteras, sino que a su vez se nutre de la emisión de los contenidos que ofrece el país con el que ha realizado el acuerdo.

\subsection{Expansión global de CCTV}

CCTV comercializó conexiones con plataformas extranjeras para emitir sus canales internacionales. Debido a la limitación de entrada de empresas extranjeras en el mercado chino, sobre todo en el sector audiovisual, las compañías extranjeras que tengan intención de intervenir en China deben realizar un joint venture con una empresa nacional; a su vez, es importante fraguar una buena relación con el régimen comunista.

En el caso de España, el canal CCTV-Español emite a través de dos portales. Por un lado, tiene un acuerdo de colaboración con la corporación pública Radio Televisión Española (RTVE) y por otro lado, un acuerdo con la empresa privada Imagenio, servicio perteneciente a la teleoperadora española Telefónica. En 2013, Imagenio desaparece adoptando el nombre de Movistar TV y se convierte en una televisión a la carta.

Actualmente, el conglomerado angloestadounidense News Corporation, fundado y dirigido por Rupert Murdoch, es la empresa extranjera con mayores privilegios en el mercado comunicativo chino.

La programación de la televisión china fuera de sus fronteras se lleva a cabo de forma mayoritaria en inglés. Para ello, CCTV ha firmado acuerdos de colaboración con Directv Group, empresa estadounidense, con el grupo estadounidense Viacom y con Sky Digital, perteneciente a British Sky Broadcasting Group, un conglomerado fundado en Reino Unido.

El canal CGTN-Français es resultado de un acuerdo realizado con el canal televisivo por suscripción France 24, perteneciente a la sociedad nacional France Médias Monde, encargada de supervisar el sector exterior audiovisual francés.

En los países de habla hispana también se percibe una influencia importante, sobre todo con Venezuela tras la aprobación de pactos mediáticos con Venevisión, perteneciente al grupo Cisneros desde 1961.

La influencia de China se extiende también hasta Rusia con la firma de acuerdos con NTV Plus, filial del grupo ruso Gazprom Media, e incluso su programación se traduce al árabe y se difunde a través de la Organización Árabe de Comunicaciones por satélite (Arabsat).

Actualmente se percibe un aumento en el interés sobre el continente africano, donde el alcance de la televisión estatal china se transmite a través del operador satelital egipcio Nilesat (Reig y Martín Ávila, 2011).

La organización de medios multiidioma y multiplataforma tal y como es CGTN no se orienta en exclusiva a la difusión de contenidos por televisión, sino que mediante una convergencia de medios en este país asiático ofrece su contenido también a través de plataformas digitales. Tiene web oficial y presencia en diversas redes sociales como por ejemplo Weibo en China y Twitter en el resto del mundo. 
El mensaje transmitido al exterior por este medio de comunicación se caracteriza por una fuerte presencia del poder blando tanto a nivel externo como interno; se realiza hincapié tradiciones culturales chinas, inspiradas en los valores confucianos, para conformar una nueva narrativa nacional que haga referencia al término acuñado por Xi Jinping de "sueño chino". De esta forma se lleva a cabo una homogeneización de la cultura televisiva.

En referencia a la utilización del lenguaje, CCTV tiende a ofrecer, tanto nacional como internacionalmente, un mensaje teóricamente objetivo y alejado de los mensajes propagandísticos de épocas pasadas. Pero la información queda en todo momento relegada a la censura y a la interferencia del poder en la comunicación.

Actualmente China se halla en su segunda estrategia comunicativa, orientada a las audiencias de América Latina y África. Esto se debe a las ansias de expansión por parte de este país asiático en busca de la transmisión internacional de la imagen y la perspectiva china. La política exterior china centra especial atención en la expansión mediática de sus contenidos, los cuales en un futuro buscan ser un referente en la comunicación respetando los valores y tradiciones chinas y combinándolos con la modernidad actual en la que está inmersa el mundo.

\section{CONCLUSIONES}

Desde la apertura China al mundo, mediante cierta liberalización del mercado, este país ha traspasado fronteras, emergiendo como una gran superpotencia en el panorama mundial. Su influencia no solo se relaciona con su poder económico, sino que la presencia de este "dragón rojo" se extiende también a nivel social en un intento de situarse como el referente del Asia-Pacífico.

La comunicación se ha convertido en un punto clave donde China está centrando gran interés; es cierto que el desarrollo de la expansión de la comunicación china es todavía un espacio incipiente, pero de forma gradual, este país asiático muestra una gran perspectiva de cambio. El Estado chino, y por consiguiente el PCCH, ha concentrado en sus manos el poder de la comunicación manteniendo un control directo sobre el mensaje; vigilar la información que se transmite a través de los diversos medios de comunicación implica de forma directa el control de la sociedad. Para ello, el PCCh ha modificado de forma continua las administraciones encargadas de las regulaciones de los medios como una respuesta a las transformaciones y la creciente complejidad que ha experimentado la sociedad china en los últimos años. La figura del poder influye en la elaboración y difusión de la información a la sociedad donde el mensaje pasa por filtros de control y adquiere un valor propagandístico, ensalzando los acontecimientos positivos y manteniendo en un segundo plano las cuestiones negativas que puedan desestabilizar el statu quo de la sociedad. China es un ejemplo de la gran fuerza que supone el control de los medios de forma partidaria.

A pesar de que las medidas de control de los medios de comunicación han sido más livianas desde las reformas impulsadas por Deng en 1978, en los últimos años se ha intensificado su presencia con la política asertiva desde la llegada al poder de Xi Jinping. La reestructuración que se ha producido en el sistema mediático estatal, tanto con la formación de un nuevo grupo mediático como es China Media Group, y las renovaciones de las administraciones censoras, suponen una vuelta a los orígenes 
de control informativo férreo producido en el gobierno de Mao Zedong donde se establece un control directo e ilimitado en referencia a los medios de comunicación y el entretenimiento del país.

Un ejemplo de esta fuerza monopolística y la relación de los medios de comunicación con el poder se ejemplifica en la televisión pública China, la cual bajo el mandato directo del PCCh ofrece contenidos basados en el soft power, mensajes oficialistas y la difusión de entretenimiento. La gran diversificación interna de este medio de comunicación muestra una característica diferenciadora con los medios de comunicación occidentales: CCTV diversifica su contenido temático por canales, donde cada canal está especializado en un tema en concreto. Esta forma de organización de contenidos se diferencia en gran medida con la programación televisiva occidental donde cada canal ofrece una gran variedad de contenidos para atraer a un mayor público.

El desarrollo de técnicas de expansión de este gigante asiático hacia el resto del mundo, buscan mantener el carácter híbrido del sistema económico y social donde los medios de comunicación juegan un papel clave en esta expansión. China ha centrado especial atención en las características de las audiencias extranjeras y en la traducción de los contenidos dependiendo del país en el que se transmitirá la información. En los últimos años los medios de comunicación chinos han orientado sus esfuerzos a establecer una sólida colaboración con zonas del mundo que antes estuvieron más relegadas como África y América Latina, así como a adaptar su contenido a Internet y las redes sociales.

Lejos ha quedado la idea global de estar ante un mundo unipolar encabezado por Estados Unidos donde solo hay un sistema válido. China no solo ha abierto sus puertas a la liberalización económica, sino que su cada vez mayor presencia en todo el mundo ha supuesto una ventana donde se muestra la variedad cultural de este país. Valores orientales, tradiciones o formas de vida se dan a conocer a nivel mundial, donde se recupera la idea de singularidad de la sociedad china basándose en los principios confucianos y sirviendo así de contrapunto al control único por parte de Estados Unidos. De esta manera, mediante la defensa de la diversidad oriental, China busca marcar un camino propio y establecer un punto de inflexión rompiendo la unipolaridad mundial y estableciéndose como un país con un sistema válido a nivel global.

De esta forma, con el paso de los años China busca tener presencia comunicativa en los cinco continentes, traspasando fronteras.

\section{Referencias (estilo ARIC_REFT)}

Aramberri, J. (2018). La China de Xi Jinping. Biblioteca Deliberar.

Borbón, A. Z. (2016). China: El despertar del dragón rojo. Boletín IEEE, (1), 901-933.

CaixaBank Research. (2017, octubre). China's Game of Thrones: the keys and complexities of the political system.

Chieh Ho, P. (2020, abril 21). Goodbye, SAPPRFT (but not Chinese censorship). SupChina. https://bit.ly/2HstqpL

China ORG. (s.f.). China's Political System. https://on.china.cn/3kkK8Gr

Council on Foreing Relations. (2019, 27 septiembre). The Chinese Communist Party https://on.cfr.org/3dJfgwM

Cunhai, G. (2017). La imagen de China en América Latina en el siglo XXI. Orientando Temas de Asia Oriental, Sociedad, Cultura y Economía, 14, 11-44. https://doi.org/10.2307/j.ctvnp0jw3.15 
Del Pozo, M. A. (2017, 27 enero). China, políticas y subconjuntos del Partido Comunista.

Delgado, L., y Ricardo, D. (2014). Confucianismo como humanidad: claves para complementar la modernidad. México y la cuenca del Pacífico, 3(9), 77-104. https://doi.org/10.32870/mycp.v3i9.472

Foix, T. A. (2011). Los periodistas políticos y los spin doctors como fuentes de la información periodística: una relación desequilibrada. En La comunicación pública, secuestrada por el mercado. Sociedad Latina de Comunicación Social, 89-90.

Guía de la Radio. (2018, 22 marzo). La República Popular China fusiona su radio y TV públicas en «Voice of China». https://bit.ly/2liGyio

Jirik, J. (2003). The Myth of the CCTV Monopoly [conferencia] Digital News, Social Change \&. Globalization, Hong Kong.

Jirik, J. (2009). The PRC's "going out" project: CCTV International and the imagination of a Chinese nation.

Madrid Morales, D., Martínez Taberner, G., y Ollé, M. (2017). Comunicación y poder en Asia Oriental. Editorial UOC.

Nelson, A. (2013). CCTV's international expansion: China's grand strategy for media? The Center for International Media Assistance (CIMA).

De Suso, B y Observatorio Iberoamericano de Asia (2007, mayo). La nueva Ley sobre el Derecho de Propiedad en China.

Ollé, M. (2007). China SL (sociedad líquida): cambio y polarización en la sociedad china contemporánea. Papeles del Este, 13, 1-21. https://doi.org/10.33115/udg_bib/cp.v6i11.22023

Ollé, M. (2017). China y medios de comunicación. L'ecosistema comunicatiu xinès: des del quiosc al panòpticon digital. Communication Papers, 6 (11), 9-26.

Organización mundial del Comercio. (2001, noviembre 23). Adhesión de la República Popular de China. https:// bit.ly/2HmfL43

Reig, R. (2000). Medios de comunicación y poder en España. Prensa, radio, televisión y mundo editorial. Paidós.

Reig, R. (2010). La telaraña mediática. Cómo conocerla, cómo comprenderla. Editorial Comunicación Social.

Reig, R. (2011). Los dueños del periodismo. Editorial Gedisa.

Reig, R., y Labio, A. (eds.) (2017). El laberinto mundial de la información. Anthropos Editorial.

Reig, R., y Martín Ávila, A. J. (2011). La comunicación en los países emergentes: estructura mediática en China, Rusia e India. Telos: cuadernos de comunicación e innovación, 87, pp. 13.

Ríos, X. (2007). Mercado y control político en China. La transición hacia un nuevo sistema. Editorial Catarata.

Ríos, X. (2016). China Moderna. Tibidabo Ediciones S.A.

Rodríguez-Jiménez, F. J. (2015). “Charm offensive?":" poder blando" chino en las últimas décadas. Pensar con la historia desde el siglo XXI [actas] XII Congreso de la Asociación de Historia Contemporánea (pp. 63916412), UAM.

Salazar Navarro, S. (2012). Del otro lado de la Gran Muralla: La transformación del sistema mediático chino (1979-2009), del modelo de propaganda a la prensa comercial. Comunicación y sociedad, (18), 55-77. https://doi.org/10.32870/cys.v0i18.191.

Sarnelli, V. (2013). "UpClose” with the Chinese soft power in CCTV News.

Sarnelli, V. (2017). UpClose, broadcasting the Chinese dream CCTV news and China's cultural policy presented to a global audience. 
Shackleton, L. (2018, abril 13). China to abolish SAPPRFT as part of cabinet restructure. ScreenDaily. https://bit. $\mathrm{ly} / 3 \mathrm{dO} 2 \mathrm{EEC}$

Tartaglione, N. (2018, abril 13). China To Ax SAPPRFT After Abolishment Of Presidential Term Limits; What's Next? Deadline. https://bit.ly/3m8vIJL

The National People's Congress of the People's Republic of China. (s.f.). Constitución de la República Popular de China. https://bit.ly/2HjSu2l

T. Rawnsley, M. Y. (2014, noviembre 14). Reflections on the Transformation of CCTV Documentary.

Xinhua. (s.f.). XIX Congreso Nacional del PCCh. Spanish Xinhua Net. https://bit.ly/34k1jCi

Xinhua. (2018, abril 16). China unveils three state administrations on film, press, television. Xinhua Net. https:// bit.ly/3obky8z

Xu, B. y Albert, E. (2014). Censura de medios en China. Consejo de Relaciones Exteriores, 25, 243.

Zhou Qian, y JAMCO Online International Symposium. (2016). China's International Television Broadcasting and Internal and External Challenges.

\section{Semblanza del autor}

Lucía Varela Monterroso es estudiante del posgrado Periodismo de Investigación Aplicado a los Medios de Comunicación en la Universidad Carlos III de Madrid. Graduada en Periodismo por la Universidad de Sevilla. Actualmente realiza una investigación académica sobre la importancia del continente asiático a nivel mundial, centrando especial atención en las razones de por qué los países asiáticos tienen menor presencia y alcance a nivel comunicativo. 